\title{
Razonamiento silogístico e interpretación de premisas: un estudio evolutivo
}

\section{Juan A. García-Madruga *}

Instituto de Ciencias de la Educación

Universidad Nacional de Educación a Distancia

La adquisición del pensamiento formal determinará la capacidad del individuo para acceder al saber científico. Y, si muchas parcelas de ese pensamiento están estudiadas desde un punto de vista evolutivo, no ocurre lo mismo con el razonamiento silogístico. Este artículo constituye uno de esos escasos estudios, del que se desprenden nuevos datos sobre las estrategias cognitivas implicadas en las diferentes edades para este tipo de tareas.

* Dirección del autor. I.C.E. Universidad Nacional de Educación a Distancia. Ciudad Universitaria. Madrid. 


\section{INTRODUCCION}

El silogismo categórico es un tipo de argumento condicional en el que el antecedente está formadó por las dos premisas, unidas mediante la con. junción y el consecuente es la conclusión. Tanto las premisas como la con. clusión son enunciados que incluyen los cuantificadores (Todo" y "Algún'), con sus negaciones (Ningún» y "Algún no). Tradicionalmente se han utilizado las letras mayúsculas A, I, E y O, para referirse a los enunciados con estos cuantificadores:
A.- "Todo A es B")
- Universal afirmativa
I.- (Algún A es B»)
- Particular afirmativa
E:- (Ningún A es B»)
- Universal negativa
O.- (Algún A no es B»)
- Particular negativa

Veamos un ejemplo:

\begin{tabular}{l} 
Todo B es C \\
Algún $A$ es $B$ \\
\hline Luego: Algún A es C
\end{tabular}

Las combinaciones de los distintos tipos de premisas y conclusión es lo que se llama modo del silogismo: el modo de nuestro ejemplo sería AI-I. En la lógica clásica el sujeto de la conclusión está en la $2 .^{\text {a }}$ premisa o premi. sa menor y el predicado en la primera o premisa mayor.

Además, el orden de los términos en cada premisa puede variar de un silogismo a otro, con lo que nos da las cuatro figuras posibles:

\begin{tabular}{|c|c|c|c|}
\hline 1. a figura & 2. ' figura & 3. afigura & 4. ' figura \\
\hline$B-C$ & $C-B$ & $B-C$ & $C-B$ \\
\hline$A-B$ & $A-B$ & $B-A$ & $\mathrm{~B}-\mathrm{A}$ \\
\hline$A-C$ & $A-C$ & $A-C$ & $A-C$ \\
\hline
\end{tabular}

La utilización del silogismo categórico para investigar el razonamiento humano ha sido constante durante todo el siglo $\mathrm{XX}, \mathrm{y}$ ha sido precisamente en el campo del razonamiento silogístico donde más claramente se ha manifestado la polémica sobre el carácter lógico o ilógico del razonamien. to humano. La característica principal del razonamiento silogístico es su gran complejidad que provoca las altas tasas de error halladas, superiores incluso a las del razonamiento condicional. Además, esta gran complejidad se manifiesta en que a pesar de la dificultad general, ésta varía ampliamente entre un silogismo y otro. Asi existen combinaciones de premisas donde la tasa de error se acerca al $100 \%$, mientras que en otras no existen practicamente errores. Nuestro ejemplo anterior (AI-I) puede ser considerado como un silogismo (fácil). Veamos una combinación de premisas de gran dificultad:

Ningún C es B.

Todo B es A

Luego: Algún A no es C. 
Un factor que influye en la dificultad del razonamiento silogístico es que de las 64 combinaciones posibles (16 combinaciones de premisas $\times \overline{4}$ figuras) más de la mitad no tienen conclusión lógicamente válida.

\section{RAZONAMIENTO SILOGISTICO E INTERPRETACION DE PREMISAS}

\subsection{Teorías y estudios del razonamiento silogístico}

La explicación más conocida de la actuación de los sujetos es la teoría del efecto (atmósfera) (Sells, 1936; Woodworth y Sells, 1935) que ha sido hegemónica durante varias décadas en el campo del razonamiento silogís. tico. Según esta teoría el rázonamiento de los sujetos está influido por la (atmósfera) de la premisas, y por tanto, la conclusión será elegida de acuerdo con esa (catmósferan o impresión global. La predicción para los silogismos con ambas premisas del mismo tipo $(\mathrm{A}, \mathrm{I}, \mathrm{E}, \mathrm{O})$ es que la conclu- . sión más frecuentemente elegida será también del mismo tipo. En los demás casos, si alguna premisa es particular existirá una tendencia a elegir una conclusión particular y si una premisa es negativa, la conclusión más frecuentemente elegida será también negativa.

El aspecto más importante del efecto (atmósfera» es que basa el razonamiento de los sujetos en un proceso de selección de los rasgos.lingüísticos superficiales de las premisas, con lo que el razonamiento seguiría un camino ajeno al de la lógica; es una toría ilógica del razonamiento. El problema fundamental del efecto (atmósfera» es que sólo se adecúa parcialmente a los resultados y que, además, está basada en unos datos experi. mentales cuya corrección metodológica ha sido puesta en entredicho.

Loren y Jean Chapman (1959) pusieron de manifiesto las debilidades del efecto (atmósfera» al mismo tiempo que propusieron una explicación alternativa. Su concepción está basada en dos hipótesis, la conversión de las premisas y la utilización por parte de los sujetos de un razonamiento probabilístico. La conversión ilícita de las premisas A y O explicaría los errores encontrados en las combinaciones en las quẹ hay conclusión válida en alguna figura y pondría de manifiesto las tendencias hacia la simetría halladas en el razonamiento proposicional. Los sujetos al convertir las premisas A y O cambian la figura del silogismo y responderian cacertada. mente». Veamos un ejemplo, la combinación AI-2:

Todo $\mathrm{C}$ es $\mathrm{B}$

Algún A es B

no tiene conclusión proposicional válida. Según la hipótesis de conversión, los sujetos convierten la premisa A cambiando la figura de silogismo y convirtiéndolo en AI-I: 
Todo B es C

Algún A es B

que como hemos vistọ tiene conclusión: Algún A es C.

Ceraso y Provitera (1971) realizaron un experimento en el que trataron de comprobar hasta qué punto los errores atribuidos al efecto atmósfera podrian ser atribuidos a una interpretación incorrecta de las premisas. Esta concepción venia avalada por el hecho de que las premisas del silogis. mo pueden referirse a diferentes relaciones entre conjuntòs. Así, cada una de las premisas puede representarse mediante diagramas de Euler (Tabla I).

TABLA 1

Diagramas que corresponden a cada premisa del silogismo

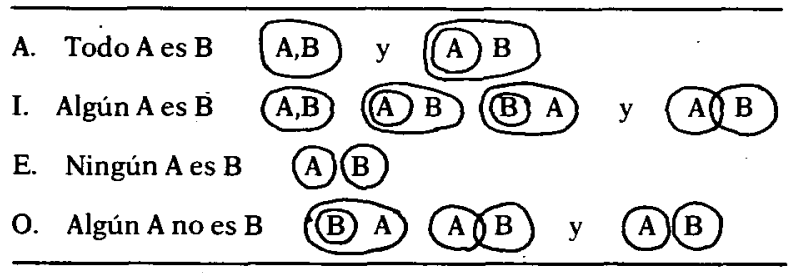

Ceraso y Provitera suponen que los sujetos simplifican la tarea y utili. zan únicamente un diagrama por premisa. Sus resultados confirmaron parcialmente las predicciones al encontrar que la presentación del problema utilizando un único diagrama eliminaba gran parte de los errores de los sujetos. No obstante, se vieron obligados a sostener que la interpretación errónea de las premisas no podría explicar totalmente la actuación de los sujetos. Era necesario también postular la existencia de una combinación parcial de los significados de las premisas. La conclusión a la que lle garon es notablemente semejante a la de los Chapman; ambas teorias sos. tienen que el razonamiento de los sujetos es, a veces, parcialmenté lógico.

El modelo de Erickson (1974, 1978), que es un desarrollo de la concepción de Ceraso y Provitera, postula la existencia de un proceso complejo en tres estadios. El primero es el de la interpretación de las premisas, el se. gundo implica la combinación de las interpretaciones realizadas y el terce. ro la elección de la forma verbal adecuada, es decir una conclusión del tipo A, E, ó O. La suposición básica es que los sujetos utilizan un tipo de representación semejante a los diagramas de Euler y propone determinados parámetros probabilísticos para cada uno de los tres estadios. Así, en el primer estadio. los sujetos escogen, según determinados parámetros de probabilidad, un sólo diagrama por premisa. En el segundo estadio los su. jetos realizan las combinaciones de los diagramas de las premisas. Tam. bién según ciertas probabilidades. Por último, en el tercer estadio la elec. ción de la forma verbal de la conclusión, que se adecúe al estadio anterior de combinación, se realiza también introduciendo parámetros probabilísti. cos. Por tanto, la actuación de los sujetos es lógica, pero parcial e incom. pleta. El modelo de Erickson plantea una importante dificultad que provie. 
ne de la utilización de los parámetros probabilísticos. Estos parámetros probabilísticos son esțimados (a posteriori), a partir de los propios datos. Además, el hecho de que estas probabilidades no hayan sido explicadas le ha permitido cambiarlas en función de sus necesidades.

El modelo de Revlis (1975 a y b) es una construcción compleja que intenta sostener la suposición básica de que el razonamiento de los sujetos es totalmente lógico. Los errores se deberían a las interpretaciones erróneas ya que los sujetos "convierten» automáticamente todas las premisas, cambiando con ello la figura de silogismo. Subsidiariamente, una segunda fuente de error surge de la resistencia de los sujetos a reconocer el hecho de que no existe conclusión proposicional válida. La operación de conversión automática se bloquearia en silogismos con contenido concreto cuan. do produce relaciones (desviadas), contrarias al conocimiento de la realidad que tiene el sujeto. Asi en el caso de premisas como "Todos los gatos son animales» la operación de conversión no se realizaria ya que el sujeto sabe que no (Todos los animales son gatos». A pesar de este matiz, el pro. blema principal del modelo de Revlis es la introducción de la conversión como una operación automática y no como fruto de una tendencia más general hacia la simetría. El modelo de Revlis, al cambiar las figuras de los silogismos, es incapaz de explicar la influencia del efecto figura en el razonamiento de los sujetos.

Dickstein (198 a y b) ha elaborado una concepción teórica en la que, además de explicar el efecto de la figura (G.2 Madruga, 1982) y desarrollar la hipótesis de la conversión de los Chapman, propone la existencia de dos procesos de error para los silogismos sin conclusión proposicional válida. En primer lugar, en las combinaciones en las que ambas premisas son particulares II, IO y OI los sujetos al considerar el término medio común llegan erróneamente a conclusiones I y $\mathrm{O}$. En el caso de las combinaciones de premisas en que ambas son negativas EE, EO, OE, y OO, la existencia de la doble negación les llevará a conclusiones $\mathrm{E}$ y $\mathrm{O}$.

El modelo analógico de Johnson-Laird (1975; Johnson-Laird y Steedman, 1978) ha supuesto una importante avance que permite explicar el efecto de la figura. En el origen del modelo están dos importantes cambios teórico-metodológicos. En primer lugar, rompió con la tradición lógica, respetada hasta entonces por los psicólogos, que obligaba a que el sujeto de la conclusión estuviera siempre en la segunda premisa. En segundo lugar, y en relación con lo anterior, Johnson-Laird ha utilizado una tarea de construcción que obliga al sujeto a elaborar su propia conclusión, sin estar limitado a las 5 alternativas de las tareas de evaluación (A, E, I, O y «No hay conclusión))).

Estos cambios le permitieron comprobar que las figuras $1\left(\begin{array}{l}\mathrm{B} \cdot \mathrm{C} \\ \mathrm{A} \cdot \mathrm{B}\end{array}\right)$ y $4\left(\begin{array}{l}\mathrm{C} \cdot \mathrm{B} \cdot \mathrm{A} \\ \mathrm{B}\end{array}\right)$ producian un sesgo especial a conclusiones A-C y C.A, respectivamente. Además, cuando los silogismos tenian conclusión válida en un sólo sentido (A.C ó C-A) la figura influia en su dificultad (G. ${ }^{a}$ Madruga, 1982).

El modelo analógico propone la existencia de un proceso heurístico por el que los sujetos llegarian a una conclusión inicial que sería compro 
bada en una fase final de prueba lógica. Si los sujetos realizan de forma completa esta fase de comprobación, sus conclusiones serán lógicamente válidas. Este proceso heurístico está fuera de la lógica y genera conclusiones iniciales del mismo tipo que alguna de las premisas coincidiendo, salvo en cuatro combinacione de premisas, con las conclusiones predichas por el efecto atmósfera.

Por otra parte, al introducir la fase de prueba lógica, el modelo permite superar las dos deficiencias fundamentales del efecto atmósfera, es decir la posibilidad de que los sujetos actúen lógicamente y el que puedan res. ponder que no hay conclusión válida. El modelo permite predecir una am. plia gama de respuestas tanto lógicas como ilógicas, con lo que explica tanto los errores principales como el que existan sujetos con una actuación totalmente correcta. Una debilidad del modelo proviene de su no inclusión de la conversión, con lo que sus predicciones de dificultad no coinciden con los resultados en los silogismos en los que ésta actúa. Al negar la converșión no puede explicar la alta dificultad del grupo de silogismos sin conclusión proposicional válida en los que, según Chapman y Chapman (1959) y Dickstein (1975, 1978a, 1978b), actúa la conversión ilicita de premisas. De esta manerà, el modelo predice para estas combinaciones de premisas: AA.2, AI-2, AI-4, AO-1, AO-4, IA-1, IA.2, OA-1 y OA.4, la misma dificultad que para las restantes combinaciones de premisas que no tienen conclu. sión proposicional válida: EE, EO, OE, OO, II, IO y OI. Los datos de Johnson-Laird y Steedman (1978) demuestran que el grupo de premisas donde actúa la conversión son mucho más difíciles que las otras combina. ciones. Los porcentajes de respuestas correctas son de $38,8 \%$ y. $80,8 \%$, res. pectivamente ${ }^{1}$.

\subsection{La interpretación de las premisas}

Dos han sido los errores en la interpretación de las premisas que han sido propuestos como explicación parcial o total de la actuación de los sujetos:

1.) Conversión ilícita: Fue propuesta como explicación de la actuación de los sujetos por primera vez por Eidens (1929) y más tarde por Chapman y Chapman (1959). La conversión sería una manifestación de una tendencia general a la simetría fomentada por factores lingüísticos y comunicativos. Además, la tendencia a la simetría, a soluciones en términos bicondiciona. les o de equivalencia, es típica del condicional. La conversión ilícita de las premisas A y $O$, ha sido defendida por numerosos autores: Agnoli (1978); Dickstein (1975, 1978a, 1978b); Mazzoco (1972); Revlin, Ammerman, Petersen y Leirer (1978); Revlin y Leirer (1978); Revlin, Leirer, Yopp y Yopp (1980); Revlis (1975a, 1975b); Wason y Johnson-Laird (1972). Otros autores, como Ceraso y Provitera (1971) y Erickson (1974, 19789) la incluyen dentro de su concepción de una manera implícita.

1 Estos porcentajes, asi como los del anterior subapartado, han sido hallados a partir de los datos de la segunda prueba del Experimento 2 de Johnson-Laird y Steedman (1978, pp. 95.98). 
Revlis y sus continuadores sostienen que los sujetos realizan la conversión de todas las premisas de una manera automática. Además, para Revlis los sujetos interpretan "Todo A es B) como (Todo B es A), mientras que para los otros autores (Todo A es $\mathrm{B}$ ), es interpretado como ( $\mathrm{A}$ es igual a B).

Con respecto a las premisas o las posiciones son menos claras. Wason y Johnson-Laird (1972) sostienen que la conversión de estas premisas se debe a la tendencia por parte de los sujetos a interpretar las premisas $O$ de forma restringida e idéntica a la de las premisas I, que sí permiten la conversión (1972, pp. 146).

2.0) Interpretación restringida de calgún. Los sujetos tienden a interpretar las premisas particulares afirmativas I, (Algún A es B) como (No todos los A son $B$ »), incluyendo el que algunos $A$ no son $B$. Asímismo, las premisas $O$, "Algún A no es B) tiendèn a ser interpretados como (No todos los A no son $\mathrm{B}$ ), con lo que algunos $\mathrm{A}$ serán $\mathrm{B}$.

Es decir, ambas premisas serian interpretadas mediante los mismos diagramas:

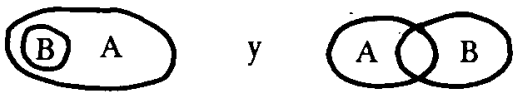

Este tipo de interpretación no lógica de las premisas particulares fue ya propuesta por Woodworth y Sells (1935) y en su versión más clara y reciente ha sido defendida, como hemos visto, por Ceraso y Provitera (1971), Erickson (1974, 1978) y Wason y Johnson-Laird (1972).

\subsubsection{Estudios sobre la interpretación de proposiciones categóricas cuantificadas.}

Neimark y Chapman (1975) realizaron un importante estudio sobre el desarrollo de la comprensión de los cuantificadores lógicos, que ha tenido gran influencia en los investigadores del razonamiento silogistico. La tarea de los sujetos consistía en verificar mediante diagramas de Euler los cuatro tipos de premisas utilizadas en el silogismo (A, E, I y O). Los resultados mostraron una mejora de la comprensión de acuerdo con la lógica, para los cuatro tipos de proposiciones. Las proposiciones universales ( $\mathrm{A}$ y $\mathrm{E}$ ) fueron interpretadas mayoritariamente de acuerdo con los cánones lógicos, mientras que las particulares ( $\mathrm{I}$ y $\mathrm{O}$ ) mantuvieron un alto porcentaje de interpretaciones restringidas, aún en los sujetos adultos universitarios. La tendencia a interpretaciones restringidas en las proposiciones particulares, fue ya encontrada por Johnson-Laird (1970) y confirmada posteriormente por Mayor (1979) y Rosenthal (1980).

Las proposiciones universales negativas (E) fueron interpretadas de acuerdo con la lógica en mayor medida que las afirmativas (A). Este resultado ha sido también corroborado por otros autores (Mayor, 1979) y no es sorprendente ya que en las proposiciones $\mathrm{E}$, los sujetos tienen que elegir un único diagrama y además coincide con los estudios del condicional en sus diferentes formulaciones. Los estudios sobre el condicional demuestran que la introducción de la negación en el consecuente $(p \rightarrow \bar{q})$, no sólo 
no aumenta la dificultad, sino que puede disminuirla (Evans, 1977; Mosham, 1980; Roberge, 1971; Wason y Evans, 1975; Wildman y Fletcher, 197.7).

En el estudio de Neimark y Chapman (1975) los sujetos más jóvenes cometían un error interesante. Interpretaban (Todo A es B) como si fuera "Todo Bं es A». Esta conversión completa es precisamente la postulada por Revlis y ha sido encontrada también en sujetos jóvenes (11.14 años) por Bucci (1978). Para esta investigación hasta los 11.12 años los sujetos suelen realizar interpretaciones pragmáticas de las proposiciones universales afirmativas. Estas interpretaciones pragmáticas suponen la no distinción entre sujeto y predicado: ((Todo A es B) $=(($ Todo, A,B). A partir de esa edad comenzarian a realizar ya interpretaciones gramaticales. La explicación aportada por Bucci, coherente con los estudios sobre el tema de Inhelder y Piaget (1959), parece más adecuada que la de Neimark y Chapman que apuntan a posibles fallos de memoria. Recientemente, Revlin y Leirer (1980) han propuesto un modelo para la tarea de verificación de proposiciones cuantificadas con diagramas, en el que aplican el modelo de conversión de Revlis. El problema fundamental de este modelo consiste en que sus predicciones no están plenamente justificadas y que, por otra parte, pueden ser explicadas de forma más sencilla sin asumir el modelo pro. puesto.

El estudio de Mayor (1979) tiene un especial interés, tanto por su amplitud como por su conexión con el razonamiento silogístico. Sus resultados aparte de comprobar con algunos matices, los presentados anteriormente, permiten elaborar un modelo de la actuación en estas tareas caracterizado por una (cvacilante ejecución de los sujetos regida, a veces, por una estrategia algoritmica (exploración serial y exhaustiva de todas las posibilidades) y, la mayoria de ellas, por una estrategia heurística (exploración de tanteo y de cortocircuitos, de ejemplos y contraejemplos)m (1979, pág. 468). Este modelo lógico reducidó viene apoyado claramente por los datos, entre los que destaca el pequeño número de errores de comisión en comparación con la notable cantidad de errores de omisión, especialmente en las premi. sas particulares. Los sujetos responden de acuerdo con la lógica, pero de una manera parcial, incompleta, simplificando la tarea a la que se enfren$\tan$.

\section{EXPERIMENTO}

\subsection{Objetivos e hipótesis}

Los objetivos del experimento son tres:

1. ${ }^{\circ}$ Comprobar los cambios con la edad en el razonamiento de los sujetos. El razonamiento silogístico ha sido un campo donde práctica. ment no ha habido estudios genéticos debido sin duda a su gran dificultad. Pensamos que un estudio que nos permita observar los cambios en la actuación de los sujetos en función de la edad, nos puede proporcionar un punto de vista enriquecedor sobre el problema. 
2.0) Comprobar en la práctica las interpretaciones que realizan los suje. tos. El recurso a las interpretaciones erróneas ha sido a nuestro entender excesivamente utilizado por algunos autores. Vamos a intentar comprobar hasta qué punto la interpretación y el razonamiento son dos procesos interrelacionados.

3. $)$ Comprobar experimentalmente los principales procesos de error en el razonamiento silogistico. Es decir, vamos a tratar de establecer las fuentes de error en el razonamiento de los sujetos que nos permitan rechazar algunas de las teorías analizadas.

Las hipótesis son:

\subsubsection{Hipótesis sobre la interpretación de proposiciones}

Se proponen dos hipótesis generales:

1. ${ }^{\circ}$ La actuación de los sujetos de acuerdo con las normas lógicas, mejorará con la edad en los cuatro niveles estudiados. Por tanto, habrá un aumento de aciertos y un descenso del número de errores en todas las proposiciones (A, E, I y O).

2.0) La actuación de los sujetos, aún los de más edad, no se adecuará com. pletamente a los cánones lógicos; es decir, será lógica pero incompleta y parcial. Por tanto, muchos sujetos omitirán algunos de los diagramas que corresponden a cada tipo de proposición. Estas omisiones se manifestarán principalmente en la tendencia a interpretar simétricamente la proposición universal afirmativa $\mathrm{A}$ y de forma restringida las proposiciones parti. culares I y $\mathrm{O}$.

Esta hipótesis permite hacer las siguientes predicciones:

a) El porcentaje de errores será muy pequeño en compáración con las omisiones, en todos los niveles de edad y para todas las proposiciones.

b) El porcentaje de respuestas simétricas para las proposiciones A será importante en todos los niveles de edad. Asímismo el porcentaje de respuestas restringidas en proposiciones I y O será importante en todos los niveles de edad.

c) El porcentaje de aciertos en todos los niveles de edad será menor en las premisas particulares que en las universales, y dentro de estas últimas será menor en las universales afirmativas (A) que en las negativas (E). Dicho de forma más sencilla, las premisas particulares serán más dificiles que las universales y las premisas A más dificiles que las $\mathrm{E}$.

\subsubsection{Hipótesis sobre el razonamiento}

Se proponen dos hipótesis generales:

1.0) Al ser el razonamiento con silogismos categóricos una tarea típica del período formal la actuación de los sujetos en la prueba de razonamien. to mejorará significativamente con la edad, en los cuatro niveles estudiados. 


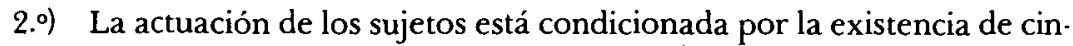
co procesos de error principales:

a) Conclusión espontánea: existe una tendencia a escoger conclusiones semejantes a alguna de las premisas que tienen conclusión válida diferente del modo de las premisas. Esta será una tendencia fuerte de error.

b) Conversión de premisas: La interpretación simétrica de las premisas A, es decir considerar (Todo A es B) como (A es igual a. B), llevará a los sujetos a conclusiones erróneas en determinadas combinaciones de premi. sas. Esta será también una tendencia fuerte.

c) Término medio común: En las combinaciones de premisas II, IO y OI los sujetos suelen considerar que el término medio es común a ambas pre. misas, con lo que eligen erróneamente conclusiones I y $\mathrm{O}$.

d) Doble negación: En las combinaciones de ambas premisas negativas EE, $\mathrm{EO}, \mathrm{OE}$ y $\mathrm{OO}$, los sujetos tienen tendencia a suponer que el sujeto y el pre. dicado de la conclusión no estarán relacionados entre sí, al no estar relacionados con el término medio en las premisas. El sujeto elegirá errónea. mente conclusiones $\mathrm{E}$ y $\mathrm{O}$.

e) Efecto de la figura: Las figuras $1 \underset{\mathrm{A} \cdot \mathrm{B}}{\mathrm{B} \cdot \mathrm{C}}$ y $4 \underset{\mathrm{B} \cdot \mathrm{A}}{\mathrm{C}}$ producen sesgos a favor de conclusiones A-C y C-A, respectivamente. Este efecto producirá mayor dificultad en combinaciones cuya conclusión es opuesta al sesgo de figura. Asímismo, en las combinaciones con conclusiones válidas en ambas direcciones se podrá comprobar este efecto.

La interpretación de estos cinco procesos de error permite hacer las si. guientes predicciones en los $32(16+16)$ silogismos que se estudiarán en este primer experimento:

1.) Existirán tres grupos de dificultad diferencial:

a) Dificultad baja: Silogismo con conclusión proposicional válida donde no actúan ninguno de los procesos de error propuestos:

$\begin{array}{ll}\text { AA.1 } & \text { AA.4 } \\ \text { EA-1 } & \text { AE-2 } \\ \text { IA.4 } & \text { AI-1 } \\ \text { AO-2 } & \text { AO-3 } \\ \text { OA.2 } & \text { OA-3 }\end{array}$

b) Dificultad media: Silogismos sin conclusión proposicional válida donde actúan los procesos de error del (término medio común» y de (doble negación):

$\begin{array}{ll}\text { II-1 } & \text { II-4 } \\ \text { IO-2 } & \text { IO-3 } \\ \text { OI-4 } & \text { OI-1 } \\ \text { OE-1 } & \text { OE-4 } \\ \text { EO-3 } & \text { EO-2 } \\ \text { EE-2 } & \text { EE-3 } \\ \text { OO-3 } & \text { OO-2 }\end{array}$


c) Dificultad alta: Silogismos donde actúan las dos tendencias de error fuertes: Conversión de premisas A y conclusión espontánea.

1) EI-4, EI-1 y IE-3 y IE-2: silogismos con conclusión válida $O$, diferente de las premisas; es decir, actúa el proceso de error de conclu. sión espontánea. Los sujetos realizan conclusiones erróneas $E$.

2) AI.4 y IA.1: Silogismos sin conclusión proposicional válida y que al convertir las premisas A llevan a conclusiones erróneas I.

e) AE-3 y EA-4 donde actúan ambos procesos de error. Estos silogis. mos serán más dificiles que el resto del grupo de alta dificultad debido a la actuación de ambos procesos de error.

2.0) El efecto de la figura se manifestará en:

a) EI-4 será más dificil que EI-1, debido a que estos silogismos tie. nen conclusión válida $O$, en la dirección A.C. La figura 4 actúa contra esta dirección de respuesta y la figura uno a favor.

b) En los silogismos con conclusión válida en ambas direcciones EA.1 y AI.1 habrá tendencia a conclusiones A.C. En IA.4 habrá ten. dencia a conclusiones C.A. En AE-2 no se predice dirección en la con. clusión.

\subsection{Método}

\subsubsection{Diseño y materiales}

Se preparó un cuadernillo con dos pruebas, una de razonamiento y otra de interpretación. El orden de presentación fue primero la prueba de razonamiento y después la de interpretación. La razón de escoger este orden secuencial, y no el contrario, se debe a que el objetivo principal del ex. perimento está en el razonamiento que realizan los sujetos y la presenta. ción previa de una prueba de interpretación (verificación con'diagramas) afectaria de manera clara la actuación de los sujetos. Por el contrario, el orden elegido permite comprobar las hipótesis siendo las interpretaciones que se realizan en la prueba de razonamiento las que pueden afectar la actuación de los sujetos en la prueba de interpretación. En un estudio piloto se presentó a 40 sujetos de $60^{\circ}$ y $8 .^{\circ}$ de E.G.B. del Colegio Siglo XXI una primera versión del cuadernillo de prueba. Este estudio piloto aconsejó reelaborar las instrucciones escritas para facilitar la comprensión de la tarea. El diseño del experimento ha sido multigrupo con repetición de las condiciones experimentales en cada uno de los cuatro grupos. El diseño puede ser considerado cuasi-experimental debido a la deficiente aleatorización.

\subsubsection{Prueba de razonamiento}

En la prueba de razonamiento se presenta a los sujetos las dos premisas de cada silogismo y se les pide que construyan ellos mismos la conclu. sión. Para evitar una gran dispersión en el tipo de respuestas, en las ins- 
trucciones se les han indicado lás caracteristicas que deben tener las posibles conclusiones. Se trata pues, de una tarea de construcción.

A cada sujeto se les presentan siguiendo un orden aleatorio, 16 combinaciones de premisas de las 64 posibles; es decir, una por cada uno de los 16 modos del silogismo y cuatro combinaciones de cada una de las cuatro figuras. Con este reducido número de items se trata de disminuir la pesadez de la prueba pensando especialmente en los sujetos más jóvenes. Para obtener datos más representativos hemos elaborado dos subpruebas de 16 ítems cada una, con un total de 32 combinaciones de premisas. Estas dos subpruebas son totalmente paralelas, de igual dificultad y variando únicamente la figura del silogismo.

Las conclusiones válidas de las 32 combinaciones de premisas pueden vérse en la tabla 2 , donde también puede observarse que cada subprueba tiene 4 items de dificultad alta; 7 de dificultad media y 5 de dificultad baja.

Los contenidos de los términos de las premisas son abstractos, usándo. se las letras B y C en la primera premisa y A y B en la segunda premisa, es . decir siendo B el término medio, y A y $\mathrm{C}$ los términos extremos.

Las instrucciones escritas previas a la prueba definen, en términos sencillos, las caracteristicas del silogismo y la tarea de los sujetos. Los ejemplos utilizados incluyen contenidos concretos y abstractos y no coinciden, en la figura, con ninguno de los ítems que tendrá que responder el sujeto. Se les explica que el término medio B no debe estar en la conclusión y se les aclara el significado lógico de (calgún», para evitar interpretaciones res. tringidas. Para evitar el sesgo contra las conclusiones no proposicionales (N), entre los ejemplos se les presenta un silogismo sin conclusión válida, y se resalta que su tarea consiste en encontrar la conclusión a los dieciseis silogismos, si es que la tienen.

TABLA 2

Conclusiones válidas de las combinaciones de premisas por subpruebas y grupos de dificultad diferencial en la prueba de razonamiento.

\begin{tabular}{|c|c|c|c|c|c|c|}
\hline & \multicolumn{3}{|c|}{ Subprueba I } & \multicolumn{3}{|c|}{ Subprueba II } \\
\hline $\begin{array}{l}\text { Dificultad } \\
\text { Alta }\end{array}$ & $\begin{array}{l}\text { AE-3.0' } \\
\text { EI-4.O }\end{array}$ & & $\begin{array}{l}\text { AI-4.N } \\
\text { IE-3.0' }\end{array}$ & $\begin{array}{l}\text { EA-4.O } \\
\text { EI-1.O }\end{array}$ & & $\begin{array}{l}\text { IA-1.N. } \\
\text { IE-2.O' }\end{array}$ \\
\hline $\begin{array}{l}\text { Dificultad } \\
\text { Media }\end{array}$ & $\begin{array}{l}\text { EE-2.N } \\
\text { II-2.N }\end{array}$ & $\begin{array}{l}\text { EO-3.N } \\
\text { OE-1.N } \\
\text { OO-3.N }\end{array}$ & $\begin{array}{l}\text { II-1.N } \\
\text { OI-4.N }\end{array}$ & $\begin{array}{l}\text { EE-3.N } \\
10-3 . N\end{array}$ & $\begin{array}{l}\text { EO-2 } \mathrm{N} \\
\text { OE-4.N } \\
\text { OO-2.N }\end{array}$ & $\begin{array}{l}\text { II-4.N } \\
\text { OI-1.N }\end{array}$ \\
\hline$\underset{\text { Baja }}{\text { Dificultad }}$ & $\begin{array}{l}\text { AA-1.A } \\
\mathrm{EA}-1_{\mathrm{F}^{\prime}}^{\mathrm{E}}\end{array}$ & OA-2.O' & $\begin{array}{l}\mathrm{AO}-2 . \mathrm{O} \\
\mathrm{IA}-\left.4\right|_{\mathrm{I}^{\prime}} ^{\mathrm{I}}\end{array}$ & $\begin{array}{l}\text { AA-4.A' } \\
\text { AE-2 }\left.\right|^{\prime \prime} \\
E^{\prime}\end{array}$ & OA-3.O & ${ }^{\mathrm{AO}-\left.1\right|_{\mathrm{I}^{\prime}} ^{I^{\prime}}}$ \\
\hline
\end{tabular}

(a) Utilizamos A, E, I y O para caracterizar la conclusión lógicamente válida en la dirección clásica A-C, y A', E', I' y $\mathrm{O}^{\prime}$ para las conclusiones en la dirección opuesta C-A. Por supuesto, las combinaciones con conclusión válida universal (A y E) tienen también conclusión válida particular (I y $\mathrm{O}$ ). Utilizamos $\mathrm{N}$, cuando no hay conclusión proposicional válida. 


\subsubsection{Prueba de interpretación}

En la prueba de interpretación se-les pide a los sujetos que verifiquen los diagramas de Euler correspondientes a cada una de las cuatro proposiciones que se les presentan. Las proposiciones son: "Todo A es C» (Algún A es $C$ ) «Ningún $A$ es $C$ ) y (Algún $A$ no es $C$ ). Los cinco diagramas se pre. sentan gráficamente a la derecha de cada una de las proposiciones y los su. jetos tienen que señalar con una cruz los que los representan correctamente. Se utilizan los términos abstractos A y $\mathrm{C}$ por coincidir con los de las conclusiones de la prueba de razonamiento. Previamente en las instrucciones escritas se. les ha recordado con palabras sencillas y fáciles, el tipo de relaciones entre los dos términos que representan cada uno de los cinco dia. gramas.

En las instrucciones escritas se informa a los sujetos de que cada una de las proporciones puede representarse por uno o varios diagramas. No se vuelve a incidir sobre el significado lógico de (algún» puesto que se pretende conocer cuáles son las interpretaciones que realizan, después de la prueba de razonamiento.

\subsubsection{Sujetos}

El número total de sujetos fue de 559, divididos en cuatro niveles de edad diferentes: $60^{\circ}$ E.G.B. $(\mathrm{N}=160$ (80 varones +80 mujeres), E:M. $=12$ años $-O$ meses), $8 .^{\circ}$ E.G.B. ( $N=160$ ( 80 V. +80 M.), E.M. $=14$ años -2 me ses), 2..$^{\circ}$ de B.U.P. $\left(\mathrm{N}=160\right.$ (80 V. +80 M.), E.M. $=16$ años -2 meses) y $1 .^{\circ}$ de Psicología ( $\mathrm{N}=79, \mathrm{E} . \mathrm{M} .=18$ años -11 meses). La elección de los niveles de edad se hizo en función de los cambios cognoscitivos que se producen a partir de los 12 años. Los sujetos universitarios fueron elegidos como grupo de sujetos adultos y la variable sexo no fue controlada, siendo mayoritariamente mujeres. En cada nivel de edad la mitad de los sujetos realiza. ron la subprueba I de razonamiento y la otra mitad la subprueba II. Todos los sujetos realizaron la misma prueba de interpretación posterior.

Los sujetos pertenecían todos a centros estatales de enseñanza, que pueden ser considerados, con algunas diferencias entre ellos, de clase media. La selección de los centros escolares no se hizo de forma aleatoria aunque sí se hizo de forma aleatoria la selección de sujetos dentro de cada centro. Asímismo, se puso especial cuidado en evitar grupos de alumnos especiales por su rendimiento escolar. Los sujetos adultos fueron de $10^{\circ}$ de Psicología de la Universidad Autónoma de Madrid y participaron en el experimento justo al principio de curso, para evitar la posible influencia de los contenidos de la asignatura de Lógica. La participación fue voluntaria aunque con las naturales limitaciones que existen en los niveles de edad más bajos.

\subsubsection{Procedimiento}

Las pruebas se realizaron colectivamente en grupos de un máximo de 40 sujetos salvo en primero de Psicología. El estudio piloto aconsejó com. 
plementar las instrucciones escritas con otras orales. Tras un primer contacto con el que se explicaba a los sujetos el sentido y objetivos de las prue. bas, tratando de motivarles adecuadamente, se les pedia que leyesen aten. tamente las instrucciones generales. Cuando todos habian leido las instruc. ciones se abría un turno de preguntas que respondia el experimentador, intentando resaltar y repetir los aspectos más importantes. En el caso de no plantearse preguntas el experimentador repetía, no obstante, estos as. pectos.

Después se les pedia que comenzasen la prueba de razonamiento y una vez terminada pasasen directamente a la de interpretación. Se midió el tiempo desde ese momento hasta el final, aunque se aclaró que debian res. ponder de forma natural y espontánea. Las pruebas se realizaron siempre a primeras horas de la mañana con los sujetos más jóvenes. El experimentador estuvo siempre ayudado por otras personas.

\section{Resultados}

\subsection{Prueba de interpretación}

Los resultados en la tarea de verificación con diagramas pueden verse en la tabla 3 y las figuras 1,2 y 3.

\section{TABLA 3}

Porcentaje de aciertos (arriba, izquierda), errores (arriba, derecha) y omisiones (abajo, cenro), por niveles de edad, en la tarea de verificación con diagramas para los cuatro tipos de proporsiciones $(A, E, I$ y $O)$.

Tipos de proposición

\begin{tabular}{|c|c|c|c|c|}
\hline & A & $\mathrm{Ea}$ & I & o \\
\hline $\begin{array}{l}\text { 6. E.G.B. } \\
\mathrm{N}=160\end{array}$ & $\begin{array}{c}15,62-38,12 \\
46,25\end{array}$ & $66,87-33,13$ & $\begin{array}{c}3,75-8,75 \\
87,5\end{array}$ & $\begin{array}{c}4,37-30 \\
65,62\end{array}$ \\
\hline $\begin{array}{l}8 .^{\circ} \text { E.G.B. } \\
N=160\end{array}$ & $\begin{array}{c}21,87-26,25 \\
51,87\end{array}$ & $88,75-11,25$ & $\begin{array}{c}3,12-3,75 \\
93,13\end{array}$ & $\begin{array}{c}3,75-25,62 \\
70,62\end{array}$ \\
\hline $\begin{array}{l}2 .^{\circ} \text { B.U.P. } \\
\mathrm{N}=160\end{array}$ & $\begin{array}{c}34,37-10,0 \\
55,62\end{array}$ & $93,12-6,88$ & $\begin{array}{c}4,37-0,62 \\
95,0\end{array}$ & $\begin{array}{c}13,12-17,49 \\
69,37\end{array}$ \\
\hline $\begin{array}{l}1 .{ }^{\circ} \text { Psicol. } \\
\mathrm{N}=78\end{array}$ & $\begin{array}{c}14,10-5,12 \\
80,77\end{array}$ & $93,58-6,42$ & $\begin{array}{c}1,27-0 \\
98,72\end{array}$ & $\begin{array}{c}3,84-10,25 \\
85,90\end{array}$ \\
\hline Total & $\begin{array}{c}22,58-22,04 \\
55,37\end{array}$ & $84,40-15,60$ & $\begin{array}{c}3,40-3,76 \\
92,83\end{array}$ & $\begin{array}{c}6,63-22,40 \\
70,96\end{array}$ \\
\hline
\end{tabular}

a) La proposición E no tiene omisiones por tener un único diagrama de respuesta correcta.

b) Un sujeto no respondió en la prueba de interpretación.

En la figura l- puede verse que el porcentaje de aciertos desciende en $1 .^{\circ}$ de Psicología en las proposiciones A y O. Sólo en la proposición E existe un aumento continuado de los aciertos con la edad $\left(\chi^{2}=53,89\right.$, g.l. $=3$, $\mathrm{p}<.0001$ ). 


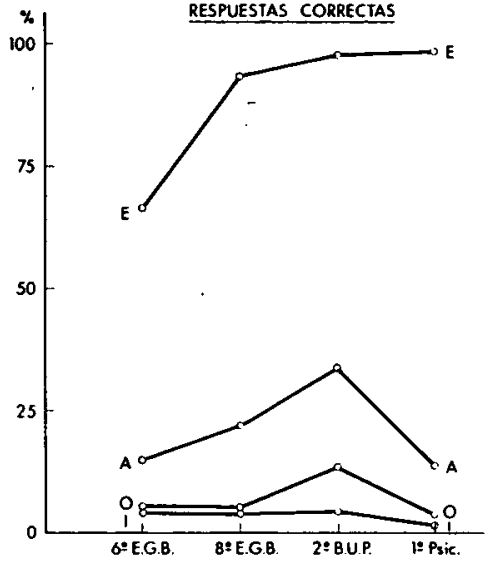

Fig. 1. Porcentaje de aciertos, por niveles de edad, de los cuatro tipos de proposición $(A, E, I, y O)$.

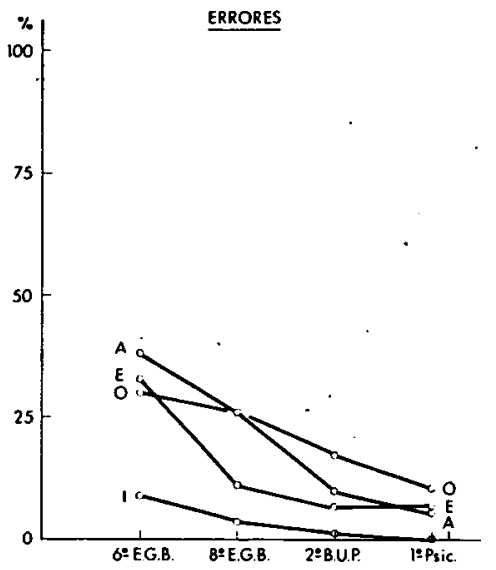

Fig. 2. Porcentaje de errores, por niveles de edad, de los cuatro tipos de proposición $(A, E, I, y O)$.

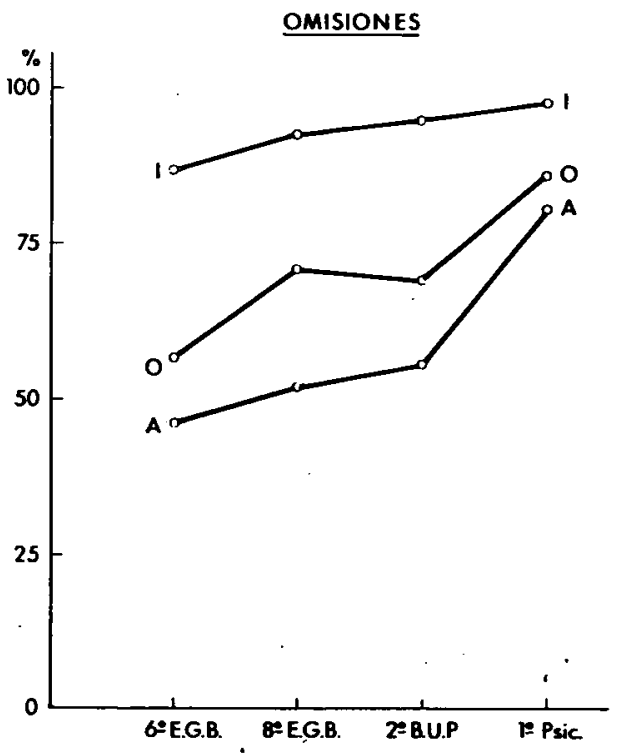

Fig. 3. Porcentaje de omisiones, por niveles de edad, en las proposiciones $A, I$ y $O$. 
En cuanto a las diferencias entre las proposiciones, las particulares I y $\mathrm{O}$ obtuvieron porcentajes de aciertos inferiores que las universales, en todas las edades. Todas las diferencias fueron significativas al nivel de .01 , menos entre las proposiciones A y O en $10^{\circ}$ de Psicología que alcanzó el nivel de .05. Asimismo, la proposición A obtuvo un porcentaje de aciertos inferior a la proposición $\mathrm{E}$, en todos los niveles de edad. Las diferencias fueron altamente significativas $(\mathrm{p}<.0001)$.

En cuanto a los errores (fig. 2), los porcentajes disminuyeron con la edad para los cuatro tipos de proposiciones (A: $\chi^{2}=56,08, \mathrm{gl}=3, \mathrm{p}<.001$; $\mathrm{E}: \chi^{2}=53,89, \mathrm{gl}=3, \mathrm{p}>.001 ; \mathrm{I}: \chi^{2}=18,36, \mathrm{gl}=3, \mathrm{p}<.001 ; \mathrm{O}: \chi^{2}=56,41$, $\mathrm{gl}=3, \mathrm{p}<.001)$.

Los porcentajes de omisiones (fig. 3) aumentan con la edad en las tres proposiciones (A: $\chi^{2}=26,5, \mathrm{gl}=3, \mathrm{p}<.001 ; \mathrm{I}: \chi^{2}=12,05, \mathrm{gl}=3, \mathrm{p}<.01$; $\left.\mathrm{O}:\left.\chi\right|^{2}=10,86, \mathrm{gl}=3, \mathrm{p}<.01\right)$. En cuanto a las diferencias entre las proposi. ciones, en la particular afirmativa I (4 diagramas) en el porcentaje de omisiones fue superior al de la particular negativa $O$ (3 diagramas) y en esta úl. tima a la de la universal afirmativa $A$. Todas las diferencias fueron signifi- cativas salvo entre O y A en $1 .^{\circ}$ de Psicología.

Los tipos de respuesta para las tres proposiciones A, I y O pueden verse en las tablas 4,5 y 6 y en las figuras 4,5 y 6 .

Los porcentajes de respuestas simétricas en la proposición A (fig. 4), varian entre $32,5 \%$ en $6 .^{\circ}$ de E.G.B. y $45 \%$ en $1 .^{\circ}$ Psicología. Las respuestas conversas erróneas tienen una pequeña incidencia, desapareciendo practicamente en $1 .^{\circ}$ de Psicología.

\section{TABLA 4}

Porcentaje de respuestas, por niveles de edad, en la tarea de verificación con diagramas para la proposición universal afirmativa $(A)$.

Tipos de respuesta a

\begin{tabular}{|c|c|c|c|c|}
\cline { 2 - 5 } \multicolumn{1}{c|}{} & No conversa & Simétrica & $\begin{array}{c}\text { Conversa } \\
\text { errónea }\end{array}$ & $\begin{array}{c}\text { Otras } \\
\text { erróneas }\end{array}$ \\
\hline 6.o E.G.B. & 29,38 & 32,5 & 14,37 & 23,75 \\
\hline 8. E.G.B. & 33,75 & 40,0 & 16,87 & 9,37 \\
\hline 2.o E.G.B. & 56,87 & 33,12 & 4,37 & 5,62 \\
\hline Psicol. & 50,0 & 44,87 & 3,84 & 1,28 \\
\hline
\end{tabular}

a) Las respuestas se han agrupado en los siguientes tipos:

No conversa: diagrama

Simétrica: diagrama

Conversa errónea: las anteriores con el diagrama

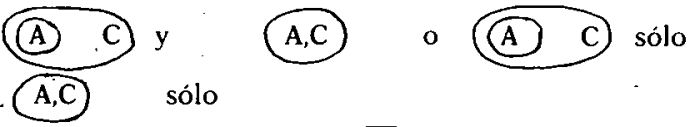

(C) $\mathrm{A}$

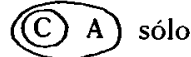


TABLA 5

Porcentaje de respuestas, por niveles de edad, en la tarea de verificación con diagramas para la proposición universal afirmativa $(I)$.

Tipos de respuesta a

\begin{tabular}{|c|c|c|c|c|}
\cline { 2 - 5 } \multicolumn{1}{c|}{} & Amplia & Restringida & $\begin{array}{c}\text { Restringida } \\
\text { Intersección }\end{array}$ & Errónea \\
\hline 6.0 E.G.B. & 53,75 & 37,5 & 26,25 & 8,75 \\
\hline 8. $^{\circ}$ E.G.B. & 60,0 & 36,25 & 31,25 & 3,75 \\
\hline 2.0 E.G.B. & 45,0 & 54,37 & 34,37 & 0,62 \\
\hline Psicol. & 30,76, & 69,23 & 42,30 & 0 \\
\hline
\end{tabular}

a) Las respuestas se han agrupado en los siguientes tipos:

Amplia: respuestas lógicamente válidas que incluyen diagramas (A,C o (A) C Restringida: los diagramas

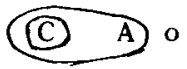
$A$ C o ambos

Restringida intersección: sólo el diagrama

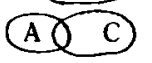

Errónea

TABLA 6

Porcentaje de respuestas, por niveles de edad, en la tarea de verificación con diagramas para la proposición particular negativa $(O)$.

Tipos de respuesta a

\begin{tabular}{|c|c|c|c|c|c|}
\hline & Amplia & Restringida & $\begin{array}{l}\text { Restringida } \\
\text { Intersección }\end{array}$ & $\begin{array}{c}\text { Conversa } \\
\text { errónea }\end{array}$ & $\begin{array}{c}\text { Otras } \\
\text { erróneas }\end{array}$ \\
\hline $6 .^{\circ}$ E.G.B. & 28,75 & 41,25 & 23,75 & 14,37 & 15,62 \\
\hline 8. ${ }^{\circ}$ E.G.B. & 26,25 & 48,12 & 27,5 & 18,12 & 7,5 \\
\hline 2. ${ }^{\circ}$ B.U.P. & 28,75 & 53,75 & 30,62 & 13,12 & 4,37 \\
\hline 1. Psicol. & 12,81 & 76,92 & 44,87 & 8,97 & 1,28 \\
\hline
\end{tabular}

a) Las respuestas se han agrupado en los siguientes tipos:

Amplia: Respuestas lógicamente válidas que incluyen el diagrama A $\mathrm{C}$

Restringidas: los diagramas

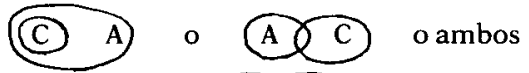

Restringidas intersección: sólo el diagrama

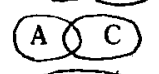

Conversa Errónea: las anteriores con el diagrama (A C o A C sólo

\section{Otras erróneas}

Las respuestas restringidas en las proposiciones I y $O$ (figs. 5 y 6) alcanzan porcentajes importantes (I: $69,23 \%$ en $1 .^{\circ}$ de Psicología; O: 76,42\% en $1 .^{\circ}$ de Psicología). Las respuestas restringidas únicas (sólo el diagrama de intersección) suponen alrededor de las $2 / 3$ partes del total de respuestas restringidas. 


\section{2}

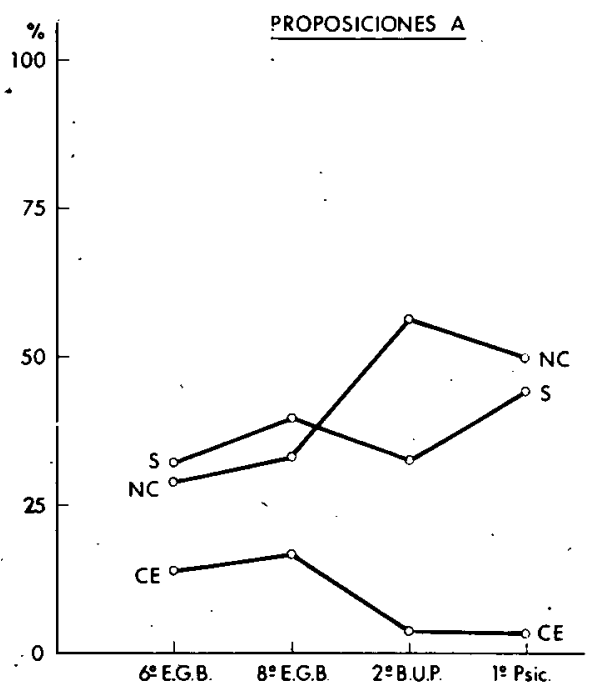

FIG. 4. Porcentaje de respuestas, por niveles de edad, para la proposición $A$.

a) N.C. $=$ No Conversa

S. $=$ Simétrica

C.E. $=$ Conversa Errónea

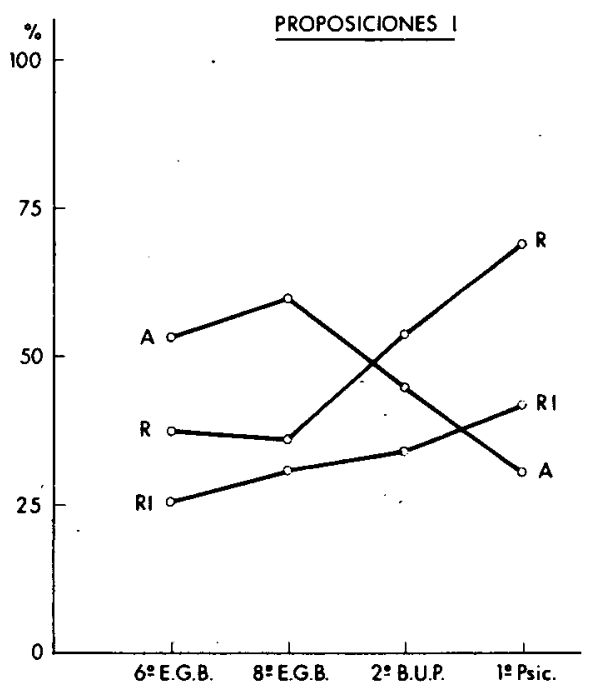

FIG. 5. Porcentaje de respuestas por niveles de edad; para la proposición I.

a) $\mathrm{A}=$ Amplia
$\mathbf{R}=$ Restringida
R.I. = Restringida Intersección

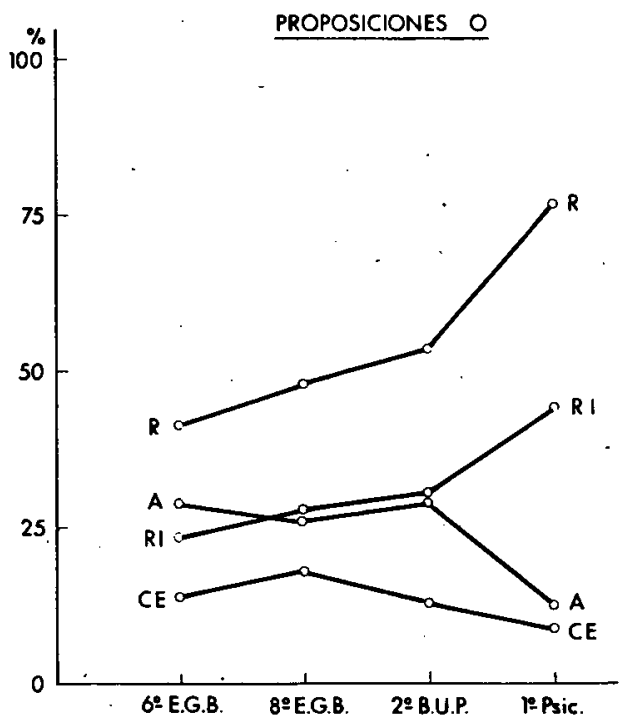

FIG. 6. Porcentaje de respuestas, por niveles de edad, para la proposición $O$.

a) $\quad A=$ Amplia

$\mathbf{R}=$ Restringida

R.I. $=$ Restringida Intersección

C.E. $=$ Conversa Errónea 
Las respuestas conversas erróneas en la proposición $\mathrm{O}$ tienen una pequeña incidencia en todas las edades, oscilando entre $18 \%$ y $9 \%$. No obstante, suponen más de la mitad del total de errores en esta proposición.

\subsection{Prueba de razonamiento}

Los resultados globales de la prueba de razonamiento pueden verse en las tablas 7 y 8 y las figuras 7 y 8 .

\section{TABLA 7}

Porcentaje de aciertos, por niveles de edad y subpruebas, en la prueba de razonamiento (Tarea de construcción)

\begin{tabular}{|l|c|c|c|}
\cline { 2 - 4 } \multicolumn{1}{c|}{} & Subprueba I & Subprueba II & Total \\
\hline 6. E.G.B. & 27,26 & 26,64 & 26,95 \\
\hline 8. $^{\circ}$ E.G.B. & 35,0 & 30,46 & 32,73 \\
\hline 2.0 B.U.P. & 40,93 & 36,87 & 38,90 \\
\hline 1.0 Psicol. & 40,38 & 40,0 & 40,18 \\
\hline Total & 35,23 & 32,56 & 33,89 \\
\hline
\end{tabular}

TABLA 8

Porcentaje de respuestas, por niveles de edad, y grupos de dificultad, en la prueba de razonamiento (tarea de construcción).

\begin{tabular}{|c|c|c|c|c|}
\cline { 2 - 5 } \multicolumn{1}{c|}{} & $\begin{array}{c}\text { Alta } \\
\text { dificultad }\end{array}$ & $\begin{array}{c}\text { Dificultad } \\
\text { Media }\end{array}$ & $\begin{array}{c}\text { Dificultad } \\
\text { Baja }\end{array}$ & Total \\
\hline 6.' E.G.B. & 3,43 & 29,91 & 41,62 & 26,95 \\
\hline 8. $^{\circ}$ E.G.B. & 5,15 & 28,92 & 60,12 & 32,73 \\
\hline 2. ${ }^{\circ}$ B.U.P. & 6,09 & 33,03 & 73,37 & 38,90 \\
\hline Psicol. & 5,69 & 34,35 & 75,94 & 40,18 \\
\hline Total & 5,00 & 31,15 & 60,85 & 33,89 \\
\hline
\end{tabular}




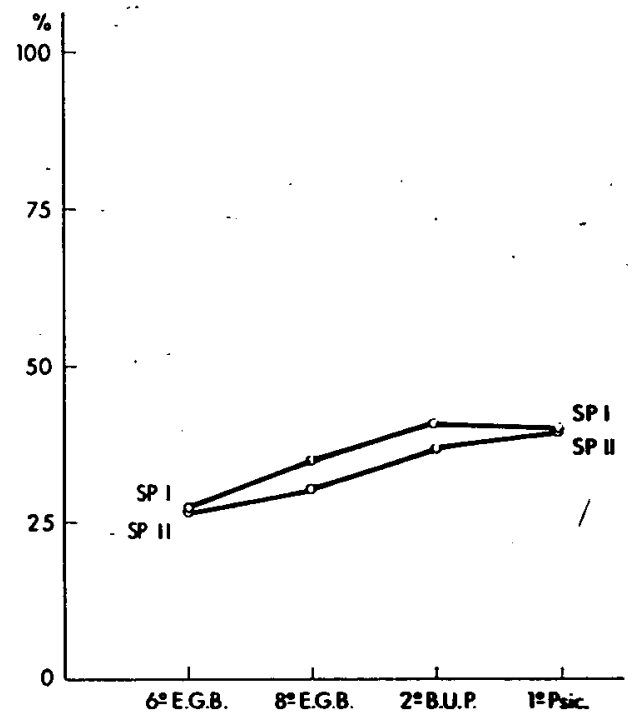

Fị. 7. Porcentaje de aciertos en la prueba de razonamiento por niveles de edad y.subpruebas.

a) S.P. I. = Subprueba I S.P. II. = Subprueba II

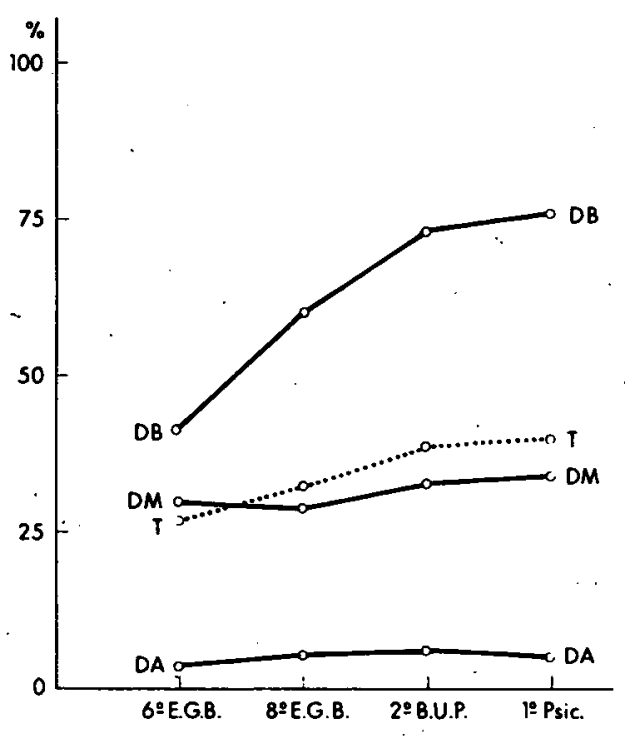

FIG. 8. Porcentaje de aciertos en la prueba de razonamiento por niveles de edad y grupos de dificultad.

a) D.B. = Dificultad baja

D.M. $=$ Dificultad media

D.A. $=$ Dificultad alta

T. = Total

- El análisis de varianza edad x subprueba (4 x 2) permitió comprobar el aumento de los aciertos con la edad $\left(F_{A}=31,83, \mathrm{gl}=3,551, \mathrm{p}<.001\right)$. Este aumento se dio en todos los niveles de edad, siendo las tres comparaciones altamente significativas $(\mathrm{p}<.001)$.

En cuanto a las dos subpruebas los resultados confirmaron en paralelismo entre ambas, aunque con una ligera diferencia ya que los sujetos realizaron más aciertos en la subprueba I que en la II, en $8 .^{\circ}$ E.G.B. y $2 .^{\circ}$ de B.U.P.. El análisis de varianza manifestó esta diferencia aunque con un nivel de significación relativamente bajo $\left(\mathrm{F}_{\mathrm{B}}=4,80, \mathrm{gl}=1\right.$ y $\left.551, \mathrm{p}<.05\right)$. Como puede observarse en la figura 8 lá interacción no fue significativa $\left(F_{B}=1,08, g l=3\right.$ y $\left.551, p>.05\right)$.

Un análisis más detallado del aumento del porcentaje de aciertos con la edad (tabla 8 y fig. 8) nos permite observar que se produce principalmen. te en el grupo de dificultad baja. En este grupo el aumento fue altamente significativo $\left(p<.001\right.$ ) en $6 .^{\circ}$ y $8 .^{\circ}$ de E.G.B. y entre $8 .^{\circ}$ de E.G.B. y $2 .^{\circ}$ de B.U.P. Entre $20^{\circ}$ de B.U.P. y $10^{\circ}$ de Psicologia la diferencia no fue significativa. En los otros dos grupos de dificultad sólo hubo aumentos significativos ( $p<.05$ ) entre $8 .^{\circ}$ de E.G.B. y $10^{\circ}$ de Psicología para el grupo de dificultad media, y entre $60^{\circ}$ de E.G.B. y $2 .^{\circ}$ de B.U.P. para el de dificultad alta.

Las diferencias en el porcentaje de aciertos entre los tres grupos de di. ficultad y en los cuatro niveles de edad fueron altamente significativas $(\mathrm{p}<.001)$. 
Los porcentajes de aciertos para cada combinación de premisas en los cuatro grupos de edad pueden verse en las tablas $9,10,11$ y 12.

En cuanto al grupo de dificultad baja se observa un menor porcentaje de aciertos en las combinaciones AO y OA. No obstante, esta diferencia disminuye con la edad.

En cuanto al grupo de dificultad alta las combinaciones de premisas donde actúan ambos procesos de error (AE-3 y EA-4) obtuvieron, en los cuatro niveles de edad, un porcentaje de aciertos significativamente inferior que el resto del grupo $(\mathrm{p}<.01)$. También se cumplió la predición sobre la mayor dificultad de la combinación EI-4 con respecto a EI-1, aunque sólo alcanzó la significación en $80^{\circ}$ de E.G.B. $(p<.01)$.

\section{TABLA 9}

Porcentaje de aciertos por combinaciones de premisas en los tres grupos de dificultad, en $6 .^{\circ}$ de E.G.B.

\begin{tabular}{|c|c|c|c|}
\hline Grupo de Baja Dificultad & \multicolumn{2}{|c|}{ Grupo de Dificultad Media } & Grupo de Dificultad Alta \\
\hline $\begin{aligned} \mathrm{AA}-1-46,25 \\
\mathrm{AA}-4-51,25 \\
\mathrm{AE}-2-52,5 \\
\mathrm{AI}-1-58,75 \\
\mathrm{AO}-2-27,5 \\
\mathrm{AO}-3-23,75 \\
\mathrm{EA}-1-47,5 \\
\mathrm{IA}-4-61,25 \\
\text { OA-2 - } 28,75 \\
\text { OA-3 - } 18,75\end{aligned}$ & $\begin{array}{l}\text { EE-2 - 27,5 } \\
\text { EE-3 - 41,25 } \\
\text { EO-2 - 32,5 } \\
\text { EO-3 - 40,0 } \\
\text { OE-1 - 31,25 } \\
\text { OE-4 - 28,75 } \\
\text { OO-2 - 35,0 }\end{array}$ & $\begin{array}{l}00-3-40,0 \\
\text { II-1 }-18,75 \\
\text { II-4 }-15,0 \\
\text { I0-2 }-41,25 \\
\text { I0-3 }-26,25 \\
0 I-1-25,0 \\
0 I-4-16,25\end{array}$ & $\begin{array}{cl}\mathrm{AE}-3- & 0 \\
\mathrm{AI}-4- & 5,0 \\
\mathrm{EA}-4- & 0 \\
\mathrm{EI}-1- & 5,0 \\
\mathrm{EI}-4- & 1,25 \\
\mathrm{IA}-1- & 10,0 \\
\mathrm{IE}-2- & 2,5 \\
\mathrm{IE}-3- & 3,75\end{array}$ \\
\hline Total: 41,62 & Tota & $: 29,91$ & Total: 3,43 \\
\hline
\end{tabular}

TABLA 10

Porcentaje de aciertos por combinaciones de premisas en los tres grupos de dificultad, en $8 .^{\circ}$ de E.G.B.

\begin{tabular}{|c|c|c|c|}
\hline Grupo de Baja Dificultad & \multicolumn{2}{|c|}{ Grupo de Dificultad Media } & Grupo de Dificultad Alta \\
\hline $\begin{array}{l}\text { AA-1- } 78,75 \\
\text { AA-4- } 62,5 \\
\text { AE-2 - } 68,75 \\
\text { AI-1 - } 75,0 \\
\text { AO-2- } 55,0 \\
\text { AO-3 - 33,75 } \\
\text { EA-1 - 72,5 } \\
\text { IA-4- } 80,0 \\
\text { OA-2 - } 28,75 \\
\text { OA-3- } 21,25\end{array}$ & $\begin{array}{l}\text { EE-2 - 52,2 } \\
\text { EE-3-33,75 } \\
\text { EO-2 - 27,5 } \\
\text { EO-3- 38,75 } \\
\text { OE-1 - 21,25 } \\
\text { OE-4- 28,75 } \\
\text { OO-2 - 38,75 }\end{array}$ & $\begin{array}{l}00-3-41,25 \\
\text { II-1 }-15,0 \\
\text { II-4 }-21,25 \\
\text { I0-2 }-31,25 \\
\text { I0-3-27,5 } \\
0 \mathrm{I}-1-21,25 \\
\text { 0I-4 }-11,25\end{array}$ & $\begin{array}{cc}\mathrm{AE}-3- & 1,25 \\
\mathrm{AI}-4- & 6,25 \\
\mathrm{EA}-4- & 0 \\
\mathrm{EI}-1- & 11,25 \\
\mathrm{EI}-4- & 1,25 \\
\mathrm{IA}-1- & 6,25 \\
\mathrm{IE}-2- & 7,5 \\
\mathrm{IE}-3- & 7,5\end{array}$ \\
\hline Total: 60,12 & Tot & 28,92 & Total: 5,15 \\
\hline
\end{tabular}


TABLA 11

Porcentaje de aciertos por combinaciones de premisas en los tres grupos de dificultad, en $2 .^{\circ}$ de B.U.P.

\begin{tabular}{|c|c|c|c|}
\hline Grupo de Baja Dificultad & \multicolumn{2}{|c|}{ Grupo de Dificultad Media } & Grupo de Dificultad Alta \\
\hline 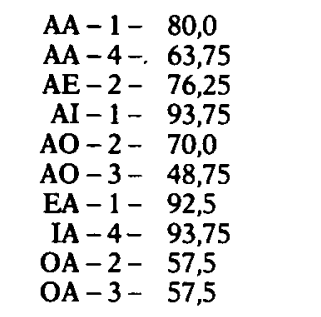 & $\begin{array}{l}\text { EE-2 - 56,25 } \\
\text { EE-3 - 45,0 } \\
\text { EO-2 - 41,25 } \\
\text { EO-3 - 31,25 } \\
\text { OE-1 - 32,5 } \\
\text { OE-4 - 28,75 } \\
\text { OO-2 - 51,25 }\end{array}$ & $\begin{array}{l}00-3-55,0 \\
\text { II-1 - } 20,0 \\
\text { II-4-13,75 } \\
\text { I0-2 - 30,0 } \\
\text { I0-3-27,5 } \\
0 I-1-15,0 \\
0 I-4-15,0\end{array}$ & \begin{tabular}{rr} 
AE - 3- & 1,25 \\
AI-4- & \multicolumn{1}{c}{5} \\
EA-4- & 1,25 \\
EI-1 - & 11,25 \\
EI-4- & 6,25 \\
IA-1 - & 6,25 \\
IE-2- & 8,75 \\
IE-3- & 8,75
\end{tabular} \\
\hline Total: 73,37 & & 35,03 & Total: 6,09 \\
\hline
\end{tabular}

TABLA 12

Porcentaje de aciertos por combinaciones de premisas en los tres grupos de dificultad, en $1 .^{\circ} \mathrm{de}$ Psicología.

\begin{tabular}{|c|c|c|c|}
\hline Grupo de Baja Dificultac & \multicolumn{2}{|c|}{ Grupo de Dificultad Media } & Grupo de Dificultad Alta \\
\hline $\begin{aligned} \text { AA-1 }-71,79 \\
\text { AA }-4-70,0 \\
\text { AE - - }-90,0 \\
\text { AI-1- } 92,5 \\
\text { AO-2- } 74,35 \\
\text { AO-3- } 60,0 \\
\text { EA-1- } 92,30 \\
\text { IA-4- } 87,17 \\
\text { OA-2- } 66,66 \\
\text { OA-3- } 55,0\end{aligned}$ & $\begin{array}{l}\text { EE-2 - 46,15 } \\
\text { EE-3 - 57,50 } \\
\text { EO-2 - 35,0 } \\
\text { EO-3 - 25,64 } \\
\text { OE-1 - 28,20 } \\
\text { OE-4- 17,5 } \\
\text { OO-2 - 60,0 }\end{array}$ & $\begin{array}{l}00-3-48,71 \\
\text { II-1 }-15,38 \\
\text { II- }-15,0 \\
\text { I0-2 - } 41,02 \\
\text { I0-3 - 42,5 } \\
0 \mathrm{I}-1-20,0 \\
0 \mathrm{I}-4-28,20\end{array}$ & $\begin{array}{cc}\text { AE - 3- } & 0 \\
\text { AI - 4- } & 2,56 \\
\text { EA - 4- } & 0 \\
\text { EI - 1 - } & 15,0 \\
\text { EI - 4- } & 7,69 \\
\text { IA - 1 - } & 2,5 \\
\text { IE-2 - } & 7,5 \\
\text { IE-3- } & 10,25\end{array}$ \\
\hline Total: 75,94 & Tot & 34,35 & Total: 5,69 \\
\hline
\end{tabular}

Los resultados con respecto a la hipótesis del efecto de la figura pue. den verse en la tabla 13.

Las predicciones sobre la dirección de la respuesta se cumplieron de manera muy clara (todas las tendencias de respuesta previstas fueron significativas). También se puede observar en la combinación AE-2 una ten. dencia no prevista a conclusiones $\mathrm{A}-\mathrm{C}$. 
TABLA 13

Porcentajes de respuestas, por niveles de edad, para las combinaciones de premisas con conclusión válida en ambas direcciones

\begin{tabular}{|c|c|c|c|c|}
\hline & $\begin{array}{c}\mathrm{AE}-\mathrm{z} \mathrm{a} \\
\mathrm{E}-\mathrm{F}^{\prime}\end{array}$ & $\begin{array}{c}\mathrm{AE}-1 \mathrm{a} \\
\mathrm{E}-\mathrm{F}^{\prime}\end{array}$ & AI-1 & $\mathrm{IA-4}$ \\
\hline $6^{\circ}$ E.G.B. & $25,0-25,0$ & $32,5-13,75$ & $41,25-17,5$ & $12,5-48,15$ \\
\hline 8. ${ }^{\circ}$ E.G.B. & $37,25-31,25$ & $52,5-18,75$ & $57,5-17,5$ & $27,5-52,5$ \\
\hline $20^{\circ}$ B.U.P. & $61,25-15,0$ & $73,75-16,25$ & $83,75-10,0$ & $12,5-81,25$ \\
\hline 1. Psicol. & $75,0-15,0$ & $69,23-23,07$ & $87,5-5,0$ & $12,82-74,35$ \\
\hline Total & $49,62-21,56$ & $56,99-17,95$ & $67,5-12,5$ & $16,33-64,21$ \\
\hline
\end{tabular}

a) Estas combinaciones tienen también como conclusión válida la proposición particular negativa, en ambas direcciones ( $\mathrm{O}$ y $\mathrm{O}^{\prime}$ ).

\section{Discusión}

\subsection{Prueba de interpretación}

La primera hipótesis sobre la mejora de la actuación de los sujetos con la edad fue totalmente confirmada en cuanto al descenso de los errores, no así en cuanto al aumento de los aciertos. En éstos el descenso para las proposiciones A y O, en $1 .^{\circ}$ de Psicologia resulta sorprendente. En la relación con este hecho está el aumento de las omisiones con la edad que no estaba previsto y contradice los resultados anteriores de Neimark y Chapman (1975), aunque es coherente con la segunda hipótesis que sostiene que la actuación de los sujetos es lógica pero imcompleta. Es decir, el número de errores lógicos es muy pequeño en comparación con el de omisiones lógicas. Además no hay que olvidar que la prueba de interpretación la realizan los sujetos después de la de razonamiento; y es posible que los sujetos de más edad tengan en la tarea de verificación de diagramas, una actuación más acorde con las interpretaciones que utilizaron en la prueba de razonamiento. En otras palabras, los sujetos de los niveles de edad superiores tienen una actuación más heurística y los de los niveles de edad inferior más algorítmica. Esta explicación del aumento de las omisiones con la edad es coherente con nuestra concepción sobre la tendencia de los sujetos a simplificar las tareas a las que se enfrentan. Por otra parte, el número de omisiones aumenta en función del número de diagramas que corresponde a cada tipo de proposición. Así, el mayor número de omisiones sé da en proposición I (4 diagramas), después en la $\mathrm{O}$ ( 3 diagramas) y por último, en la A (2 diagramas). Estos resultados confirman los de Mayor (1979) y apoyan la propuesta de Revlis (1975a). 
Otro aspecto interesante que parecen mostrar los resultados encontrados es el hecho de que la comprensión de las proposiciones A y O plantea dificultades especiales que se manifiestan en un descenso más tardio de los errores. La explicación está en el hecho de que estas proposiciones no per. miten la conversión lícita y, tal como vimos los sujetos más jóvenes tienen tendencia a interpretar estas premisas intercambiando el sujeto y el predicado, de una forma pragmática y no gramatical (Bucci, 1978). Esta explicación viene corroborada por la existencia de los errores de conversión, específicos de estas proposiciones, por su disminución con la edad. En particular, los resultados parecen mostrar que la proposición que más problemas plantea es la particular negativa $O$ ya que, aún entre los sujetos adultos, siguen existiendo errores de conversión.

Los aciertos son precisamente las respuestas sin errores ni omisiones, es decir las respuestas totalmente lógicas. Los resultados confirman la predicción de la segunda hipótesis sobre la dificultad diferencial de las proposiciones. Las particulares ( $\mathrm{I}$ y $\mathrm{O}$ ) son más difíciles que las universales, y dentro de estas últimas la afirmativa.(A) es más difícil que la negativa (E). Estos resultados son también comunes a Neimark y Chapman (1975) y Mayor (1979).

Las otras dos predicciones de la segunda hipótesis se cumplieron también de forma clara. Los sujetos demuestran una actuación lógica pero im. completa y parcial al cometer pocos errores y una notable cantidad de omisiones, en particular de respuestas simétricas (A) y restringidas ( $\mathrm{I}$ y $\mathrm{O}$ ).

En cuanto a las respuestas "conversas erróneas) hay que destacar que los resultados vienen a falsar la hipótesis de Revlis sobre la conversión au. tomatica. El porcentaje de respuestas conversas erróneas en la proposición A es muy pequeña en todas las edades alcanzando un nivel mínimo en los sujetos adultos $(3,84 \%$ ). Én cuanto a la proposición particular negativa $O$, la incidencia de las conversas erróneas, aunque algo superior a la existente en la proposición A, tiene una importancia minima entre los sujetos adul. tos $(8,97 \%)$.

\subsection{Prueba de razonamiento}

Los resultados presentados en el apartado anterior confirman de forma clara las dos hipótesis propuestas en la tarea de razonamiento silogistico.

La primera hipótesis sobre la mejora de la actuación con la edad se manifestó en las tres diferencias de niveles de edad. El aumento más importante en el porcentaje de aciertos se dio entre $8 .^{\circ}$ de E.G.B. y $2 .^{\circ} \mathrm{de}$ B.U.P. $(32,75 \%-38,90 \%)$ y el menor entre $2 .^{\circ}$ de B.U.P. y $1 .^{\circ}$ de Psicología $\left(38,9 \%-40,18 \%\right.$ )., Sin embargo, con respecto a los sujetos de $6 .^{\circ}$ de E.G.B. es posible que el porcentaje de aciertos esté artificialmente aumentado de. bido a una incidencia excesiva de conclusiones no proposicionales. Entre estos sujetos más jóvenes existió una tendencia a huir de la tarea, bien por su incomprensión previa, bien por su complejidad excesiva que se mani- 
fiesta en el alto porcentaje de respuestas erróneas no clasificables $(14,8 \%)$. Asimismo, es bastante probable que estos sujetos más jóvenes utilizasen también la respuesta (No hay conclusión) como otra forma de huir de la tarea, con lo que el número de aciertos en las combinaciones sin conclusión proposicional válida aumenta artificialmente. No hay que olvidar que de las 32 combinaciones de premisas 16 no tienen conclusión proposicional válida. Una confirmación de esta propuesta está en el descenso con la edad del porcentaje de conclusiones po proposicionales con respecto al total de aciertos, y en particular en las primeras edades.

Esta propuesta se manifiesta también, en que en el grupo de dificultad media, en el que los silogismos no tienen conclusión proposicional válida, el porcentaje de aciertos en superior en $6 .^{\circ}$ de E.G.B. $(29,91 \%)$ que en $8 .^{\circ}$ de E.G.B. $(28,92 \%)$.

En cuanto al grupo de primero de Psicología, que demostró un aumen: to significativo pero pequeño con respecto a $2 .^{\circ}$ de B.U.P, los resultados deben ser tomados con prudencia. Hay que señalar que tanto el diferente nú. mero total de sujetos, como las características específicas de estos y el hecho de que la prueba fuese pasada a todos los sujetos a la vez, limitan la generabilidad de los resultados. En particular es posible que la mejora en la actuación en los sujetos adultos sea mayor que la encontrada en este expe. rimento, como Piaget (1970) predice para las tareas formales.

\section{TABLA14}

Porcentajes, por niveles de edad, de respuestas erróneas no clasificables y de aciertos en combinaciones de premisas sin conclusión proposicional válida con respecto al total de aciertos.

\begin{tabular}{|c|c|r|}
\cline { 2 - 3 } & \multicolumn{1}{c|}{$\begin{array}{c}\text { Respuestas erróneas } \\
\text { no clasificables }\end{array}$} & $\begin{array}{c}\text { Aciertos en no válidos } \\
\text { respecto al Total }\end{array}$ \\
\hline 6. $^{\circ}$ E.G.B. & 14,8 & 50,86 \\
\hline $8^{\circ}$ E.G.B. & 7,85 & 39,85 \\
\hline 2. $^{\circ}$ B.U.P. & 2,92 & 38,05 \\
\hline 1.० Psicol. & 3,24 & 37,79 \\
\hline
\end{tabular}

En resumen, la mejora de la actuación con la edad queda compro. bada de forma clara e irrefutable, pudiendo ser, si acaso, superior a la mos. trada por los resultados encontrados.

Las diferencias en el porcentaje total de aciertos, a favor de la subprue. ba $\mathrm{I}$, entre ambas subpruebas se reducen únicamente a dos niveles de edad, 8. ${ }^{\circ}$ de E.G.B. y 2..$^{\circ}$ de B.U.P., y tienen un nivel de significación relativamente bajo (.05). Este nivel puede ser rechazado debido a la deficiente aleatorización y el número alto de sujetos. Este rechazo viene confirmado 
por el hecho de que el porcentaje de respuestas erróneas no clasificables, que denotan incomprensión o huida de la tarea, es claramente superior en la subprueba II que en la $I$.

En $80^{\circ}$ de E.G.B. el porcentaje de respuestas erróneas no clasificables en la subprueba I fue de 5,25, y en la prueba II de 10,47. En 2. ${ }^{\circ}$ de B.U.P., en la subprueba I fue de 1,32, y en la subprueba II de 4,53.

Las predicciones de la segunda hipótesis sobre los cinco procesos de error, fueron ampliamente confirmadas por los resultados. En el grupo de dificultad baja y, principalmente, en los niveles de edad inferiores, los por. centajes de aciertos en las combinaciones de premisas AO y OA fueron menores que los del resto del grupo. Estos resultados pueden tener dos explicaciones fundamentales. En primer lugar, la especial dificultad de las proposiciones o (premisas y conclusión) en los sujetos más jóvenes. Esta explicación está confirmada por el mayor porcentaje de error encontrado en la proposición $\mathrm{O}$ en la tarea de verificación con diagramas. Tal como vimos, las dificultadas de comprensión de esta proposición se deben a que une la ambigüedad del significado de (calgủn» con el hecho de tener conversión ilícita.

En segundo lugar, estas combinaciones de premisas son las únicas que dentro del grupo de dificultad baja, tienen una única conclusión válida (AO-2.0, AO-3.0, OA-2.0, OA-3.0) con lo que es normal que el porcentaje de aciertos sea inferior.

En el grupo de dificultad media los resultados confirmaron su diferencia significativa con respecto al de dificultad baja.

En el grupo de dificultad alta los resultados también confirmaron las predicciones. La diferencia respecto al grupo de dificultad media fue siempre altamente significativa. La diferencia entre las combinaciones de premisas donde actúan dos procesos de error principales (AE-3 y EA-2) y el resto del grupo fue siempre significativo. La predicción del efecto figura en cuanto al mayor porcentaje de aciertos en EI.l que en EI-4 se confirma también aunque la diferencia fue sólo significativa en $8 .^{\circ}$ de E.G.B. No debe sorprender el que estas diferencias no fueran significativas ya que se refieren sólo a un par de combinaciones de premisas.

En las combinaciones de premisas donde actúa únicamente el proceso de error de conversión (AI-4 y IA-1), los resultados demuestran que los sujetos caen mucho más en este error en la prueba de razonamiento que en la tarea posterior de verificación con diagramas.

Los resultados confirman la idea de que el tipo de interpretación que realizan los sujetos en una tarea de razonamiento está condicionado por las características y dificultades específicas que plantean la tarea, y serán, por tanto, diferentes de las interpretaciones realizadas en la tarea de verifi. cación. Hay dos aspectos importantes en estos resultados, por una parte la comprobación de que muchos sujetos que cometen errores en la prueba de razonamiento al simplificar la tarea más allá de lo que permitan las re. glas lógicas, demuestran una competencia lógica superior en la tarea de 
verificación. Por otra, el hecho de que los modelos secuenciales no pueden dar cuenta cabal de la compleja actuación de los sujetos a no ser que introduzcan mecanismos de interacción. Es decir, muchos sujetos enfrentados a la prueba de razonamiento pueden interpretar, en un primer estadio, adecuadamente las premisas, pero la búsqueda de la solución las va a llevar a una reinterpretación de las premisas que les permita garantizar la conclusión espontánea.

\section{TABLA15}

Porcentajes de aciertos, por niveles de edad, en las combinaciones de premisas Al-4 y IA-1 de la prueba de razonamiento y de respuestas no conversas en la tarea de verificación con diagramas.

\begin{tabular}{|c|c|c|}
\cline { 2 - 3 } \multicolumn{1}{c|}{} & $\begin{array}{c}\text { Prueba de razonamiento } \\
\text { (AI-4+IA-1) } \\
\text { Aciertos }\end{array}$ & $\begin{array}{c}\text { Tarea de verificación } \\
\text { Respuestas no conversas }\end{array}$ \\
\hline 6.' E.G.B. & 7,5 & 29,38 \\
\hline 8. E.G.B. & 16,25 & 33,75 \\
\hline 2.0 B.U.P. & 5,62 & 56,87 \\
\hline 1.o Psicol. & 2,53 & 50,0 \\
\hline Total & 5,47 & 42,5 \\
\hline
\end{tabular}

En cuanto al efecto de la figura se confirmaron todas las predicciones, con la particularidad de encontrar una tendencia hacia respuestas $A-C$ en $A E-2$. Este hecho podría ser explicado mediante una reformulación de la hipótesis del efecto figura ( $G^{\mathbf{a}}$ Madruga, 1982).

\section{Conclusión}

En un apartado anterior propusimos como objetivos de este primer experimento el estudio de los cambios con la edad en la actuación de los sujetos, la comprobación de los procesos de error propuestos en el razonamiento y el estudio de las relaciones entre interpretación y razonamiento. Las hipótesis propuestas hạ sido ampliamente confirmadas aunque con algunos matices que son especialmente significativos. Uno de estos matices es que además de comprobar sólo parcialmente la mejora de la actuación con la edad, en la tarea de verificación con diagramas, existe al mismo tiempo un aumento de las omisionés, es decir, un aumento de las interpretaciones parciales e incompletas. Los sujetos de los niveles de edad más altos, al menos en la tarea de verificación, simplifican más la tarea, tienen una actuación más heuristica, menos algorítmica que los más jóvenes. 
En cuanto a la relación entre interpretación y razonamiento podemos concluir que la actuación de los sujetos muestra una tendencia general simplificadora, una estrategia de simplificación de la tarea. Esta estrategia se manifiesta tanto en los tipos de error que cometen los sujetos en la prueba de razonamiento como en las interpretaciones parciales y restringidas de la tarea de verificación con diagramas. Ahora bien,-los resultados demuestran que la actuación de loś sujetos depende de la tarea a la que se enfrentan, de su nivel de dificultad. Los sujetos simplifican más las interpretaciones en la tarea de razonamiento como manifestación de una tendencia a la reducción del ésfuerzo cognitivo. (Bruner, Goodnow y Austin, 1956, p. 114). Este aspecto pone en cuestión los modelos secuencialeslineales ya que muestra que las fases de interpretación o codificación y de combinación o inferencia están mutuamente interrelacionadas y que su separación, favorecida por la metáfora computacional, es principalmente analítica.

Por último, los resultados obtenidos en la prueba de razonamiento se adecúan de forma especialmente satisfactoria a los cinco procesos de error propuestos. Esto supone que en el razonamiento silogístico existe además del proceso general de simplificación de la interpretación o de la combina. ción (Conversión de premisas, Término medio común y Doble negación) un proceso de codificación lingüística superficial semejante al «efecto atmósfera') y que explicaria la tendencia a las conclusiones espontáneás.

\section{Referencias}

AGNOLI, F. (1978). Content and Structure in Syllogistic Reasoning. Italian Journal of Psychology, Vol. 5, 3, 245-259.

Bruner, J. S., Goodnow, J. J. y Austin, G. A. (1956). A Study of Thinking, Nueva York, Wiley. Trad. es. pañola: El proceso mental en el aprendizaje. Ed. Narcea, Madrid, 1978.

BuCCI, E. (1978). The interpretation of universal affirmative propositions. Cognition, vol. 6 (1), 55.77.

Ceraso, J. y Provitera, A. (1971). Sources of error in Syllogistic reasoning. Cognitive Psychology, 2, 400-410. Trad. española en Delval, J. A. (ed.). Investigaciones sobre lógica y psicologia Alianza Univer sidad, Madrid, 1977.

Chapman, L. J. y Chapman, J. P. (1959). Atmosphere effect reexamined. Journal of Experimental Psychology, 58, 220-226. Trad. española en Delval, J. A. (ed.) Investigaciones sobre lógica y Psicología. Alianza Universidad; Madrich 1977.

DiCKSTEIN, L. S. (1975). Effect of instructions and premise order on errors in Syllogistic reasoning. Journal of Experimental Psychology: Human Learning and Memory, 1, 376.384.

DiCKSTEIN, L. S. (1978 a). The effect of figure in syllogistic reasoning. Memory and Cognition, 6, 76.83.

DiCKSTEIN, L. S. (1978 b). Error processes in syllogistic reasoning. Memory and Cognition, 6, 537.543.

EIDENS, H. (1929). Experimentalle Untersuchungen über den Denkverlauf bei unmittelbaren Folge. rungen. Archiv für die Gesamte Psychologie, 71, 1.66.

ERICKSON, J. R. (1974). A set analysic theory of behavior in formal syllogistic reasoning tasks. En Solso, R. L. (ed.) Theories of cognitive psychology: The Loyola Symposium. Nueva Jersey: Lawrence Erlbaum.

ERICKSON, J. R. (1978). Research on Syllogistic Reasoning. En R. Revlin y R. Mayer (eds.). Human Reasoning. Washington D.C.: Winston. 
Evans, J. St. B. T. (1977). Lingüistic factors in reasoning. Quaterly Journal of Experimental Psychology, 29, 297.306.

G.' MAdruga, J. A. (1982). Un estudio sobre el efecto de la figura en el razonamiento silogistico. Estudios de Psicología (en prensa).

INHELDER, B. y PIAGET, J. (1959). La genèse des structures logiques elementaires. Classifications et sériations. Neuchâtel: Delachaux y Niestlè. Trad. castellana de M. Riani: La génesis de las estructuras lógicas elementales. B. Aires: Guadalupe, 1967.

Johnson.LaIRD, P. N. (1975). Models of deductions. En R. J. Falmagne (ed.): Reasoning: Representation and Process Nueva Jersey: Lawrence Erlbaum.

Johnson.Laird, P. N. y Steedman, M. (1978). The Psychology of Syllogisms. Cognitive Psychology, 10, 64.99.

MAYOR, J. (1979). La verificación de las premisas como base del razonamiento silogístico. Revista de Psicología General y Aplicada, 34,415.472.

MAzzocco, A. (1972). Due experimenti sul ragionamiento deduttivo: il problema della conversa. Revista de Psicologia, 66, 47.65.

MOSHMAN, D. (1980). Representation and process in reasoning about logical relationshpis. The Journal of General Psychology, 103, 251.262.

NeimarCK, E. D. y ChapMan, R. H. (1975). Development of the comprehension of logical quantifiers. En: R. J. Falmagne (ed.): Reasoning: Representation and Process, Nueva Jersey: Lawrence Erlbaum.

PIAGET, J. (1970). L'evolution intellectuelle entre l'adolescence et l'age adulte. Rapport sur le III Congrès Internationale FONEME, Milán. Trad. cast. en J. A. Delval (ed.): Lecturas de Psicología del niño. Tomo 2, Madrid, Alianza Editorial, 1978.

Reviln, R.; AMmERMan, K.; PETERSON, K. y LeIRER, V. (1978). Category relations and syllogistic reasoning. Journal of Educational Psychology, 70, 613.625.

REVLIN, R. y LeIRER, V: (1978). The effects of personal biases on syllogistic reasoning: Rational decisions from personalized representations. En R. Revlin y R. Mayer (eds.): Human Reasoning. Washington: Winston.

REVLIN, R.; LEIRER, V;; YOPP, H. y YOPP, R. (1980). The belief-bias effect in formal reasoning: The in. fluence of knowledge on logic. Memory and Cognition, 8, 584.592.

REVLIS, R. (1975 a). Syllogistic reasoning: Logical decisions from a complex data base. En R. Falmagne (ed.): Reasoning: Representation and Process. Nueva Jersey: Lawrence Erlbaum.

REVLIS, R. (1975 b). Two models of syllogistic reasoning: Feature selection and conversion. Journal of Verbal Learning and Verbal Behavior, 14, 180.195.

ROBERGE, J. J. (1971). An analysis of response patterns for conditional reasoning schemes. Psychonomic Science, 22,$338 ; 339$.

ROSENTHAL, D. J. A. (1980). Logical quantifiers in affirmative and negative contexts. The Journal of General Psychology, 102, 99.106.

SELLS, S. B. (1936). The atmosphere effect: an experimental study of reasoning. Archives of Psychology, $29,3.72$.

WASON, P. C. y EVANS, J. St. B. T. (1975). Dual processes in reasoning. Cognition, 3, 141-154.

WASON, P. C. Y JOHNSON.LAIRD, P. N. (1972). Psychology of reasoning: structure and content. London, Bats. ford. Trad. cast. de J. A. Delval: Psicología del Razonamiento, Madrid, ed. Debate, 1980.

WILDMAN, T. M. y FLETCHER, H. S. (1977). Developmental increases and decreases in solutions of con. ditional syllogisms problems. Developmental Psychology, 13, 630.636.

WOODWORTH, R. S. y SELLS, S. B. (1935). An atmosphere effect in formal syllogistic reasoning. Journal of Experimental Psychology, 18, 451-460. Trad. cast. en J. A. Delval (ed.): Investigaciones sobre lógica y psicología, Alianza, Madrid, 1977. 
Resumen

Los errores en el razonamiento silogístico han sido explicados bien en términos de interpretaciones y combinaciones erróneas de los significados de las premisas, bien a causa de sesgos de respuestas producidos por los rasgos lingüisticos superficiales de las premisas. En el presente estudio se postula la actuación de ambos tipos de factores. En el experimento se le presentan a sujetos de cuatro niveles de edad diferente una tarea de razonamiento silogístico, seguida de otra tarea de verificación de premisas mediante diagramas de Euler. Los resultados muestran, con algunos matices, una mejora en la actuación de los sujetos con la edad en ambas tareas. Asimismo confirman las hipótesis planteadas tanto en la prueba de razonamiento como en la de interpretación. Estos resultados suponen una crítica a los modelos secuenciales lineales que ignöran la interacción entre las fases de codificación e inferencia. Asimismo apoyan la existencia de un proceso general de simplificación de la tarea que, junto con la tendencia a conclusiones respontáneasw, debidas a una codificación lingüistica superficial, explicarín los errores de los sujetos.

\section{Résumé}

Les erreurs dans le raisonnement syllogistique ont été expliquées soit dans des termes d'interprétations et de combinaisons erronées des signifiés des prémises, soit par des biais de réponse produits par les traits linguistiques superficiels des prémises. Dans la présente étude on postule l'action de ces deux types de facteurs. Notre expérience présente à des sujets de quàtre niveaux d'äge différent une tâche de raisonnement syllogistique, suivie par une au'tre tâche de vérification de prémises par des diagrammes d'Euler. Les resultats montrent, avec quelques nuances, dans les deux tâches, une amélioration dans le comportement des sujets avec l'äge en même temps qu'ils confirment les hypothèses posées dans la preuve de raisonnement ainsi que dans celle d'interprétation. Ces résultats supposent une critique des modèles séquenciels linéaires qui ignorent l'interaction entre les phases de codification et d'inférence. En même temps ils appuient l'existence d'un procesus général de simplification de la tâche qui, â côté de la tendance vers des conclusions aspontanéess, dües à une codification linguistique superficielle, expliqueraient les erreurs des sujets.

\section{Summary}

The errors in syllogistic reasoning have been explained either by the erroneous interpretations and combinations of premise meanings, or by the response bias made by the superficials linguistics features of the premises. The present paper sets that both factors are acting. In this experiment, subjects of four different age levels have been tested in a Euler's diagrams verification of premises task, followed by a syllogistic reasoning task Significant improvement of performance with age was found, and the hypothesis were confirmed in both tasks. The results suppose to be a critic for.the sequential-lineal models that deny the interaction betwen codification and inference phases. Likewise, they support an explanation in terms of two error processes: a general process of simplification of task and a tendency to espontaneouss conclusions, made by a superficial linguistic codification. 